\title{
Magnetic resonance imaging of adrenal gland: state of the art
}

\author{
Fabiano Vito d'Amuri ${ }^{1}$, Umberto Maestroni ${ }^{2}$, Francesco Pagnini ${ }^{1}$, Umberto Russo ${ }^{1}$, Elisa Melani ${ }^{2}$, \\ Francesco Ziglioli ${ }^{2}$, Giulio Negrini ${ }^{1}$, Simone Cella ${ }^{1}$, Salvatore Cappabianca ${ }^{3}$, Alfonso Reginelli ${ }^{3}$, \\ Antonio Barile ${ }^{4}$, Massimo De Filippo ${ }^{1}$
}

${ }^{1}$ Department of Medicine and Surgery, Unit of Radiologic Science, University of Parma, ${ }^{2}$ Department of Medicine and Surgery, Unit of Urology, Maggiore Hospital, Parma, Italy; ${ }^{3}$ Department of Radiology and Radiotherapy, University of Campania "Luigi Vanvitelli”, Napoli, Italy; ${ }^{4}$ Department of Biotechnological and Applied Clinical Sciences, University of L'Aquila, Ospedale San Salvatore, L'Aquila, Italy

Contributions: (I) Conception and design: All authors; (II) Administrative support: All authors; (III) Provision of study materials or patients: All authors; (IV) Collection and assembly of data: All authors; (V) Data analysis and interpretation: All authors; (VI) Manuscript writing: All authors; (VII) Final approval of manuscript: All authors.

Correspondence to: Francesco Pagnini. Department of Medicine and Surgery, Unit of Radiologic Science, University of Parma, Maggiore Hospital, Via Gramsci 14, Parma, Italy. Email: f.pagnini90@gmail.com.

\begin{abstract}
Detection of adrenal lesions, because of the widespread use of imaging and especially highresolution imaging procedures, is increased. Because of the importance to characterize those findings, magnetic resonance imaging (MRI), in particular chemical shift imaging (CSI), is useful to distinguish whether a lesion is benignant or malignant and to avoid further diagnostic or surgical procedures. It represents the first choice of imaging in patient like children or pregnant women, and a valid complement to other imaging techniques like CT or PET/CT. In this review we analyze the role and characteristic of MRI and the imaging features of most common benignant (adenoma, hyperplasia, pheochromocytoma, hemorrhage, cyst, myelolipoma, teratoma, ganglioneuroma, cystic lymphangioma, hemangioma) and malignant [neuroblastoma, adrenocortical carcinoma (ACC), metastases, lymphoma] adrenal lesions.
\end{abstract}

Keywords: Imaging; magnetic resonance imaging (MRI); adrenal glands

Submitted Jan 28, 2019. Accepted for publication Jun 03, 2019.

doi: $10.21037 /$ gs.2019.06.02

View this article at: http://dx.doi.org/10.21037/gs.2019.06.02

\section{Introduction}

The adrenal glands are a frequently involved site of disease of the general population. Adrenal lesions are often an incidental finding as the use of cross-sectional imaging is increased. When adrenal masses are detected is crucial to discriminate benign from malignant lesions and find out whether the lesion is primary or secondary. The assessment of adrenal disorders can effectively rely on magnetic resonance imaging (MRI) and on its tissue characterizing ability (1). The physiological aspect of adrenal glands, on T1- and T2-weighted imaging, is characterized by low to intermediate signal intensity. Cystic changes, variation in vascularity or hemorrhage may be seen within an adenoma as small, rounded foci of altered signal intensity. Chemical shift imaging (CSI) is the cornerstone of adrenal MRI.
The chemical shift phenomenon relies on the differential precession frequencies of protons in lipid and water within the same imaging voxel. The loss of signal intensity on outof-phase breath-hold gradient-echo MRI when compared with in-phase images, depends on the lower precession frequency of fat protons compared to water protons (2). Many adenomas and especially lipid-rich type show high signal intensity on in-phase imaging and low signal intensity on out-of-phase imaging, allowing recognition on CSI by simple visual assessment (3). The use of splenic intensity on CSI as a reference is the most common method to analyze an adrenal lesion. In lipid-rich adenomas, where the concentration of fat and water protons are equivalent, there is almost complete signal intensity loss on out-ofphase images. Otherwise, in lipid-poor adrenal adenomas, there are no changes of signal intensity on out-of-phase 
Table 1 Suggested protocol for MRI study of adrenal glands

\begin{tabular}{lcc}
\hline Sequence type & Imaging plane & Section thickness $(\mathrm{mm})$ \\
\hline T2-weighted & Axial and coronal & 5 \\
T2- weighted fat-suppressed & Axial & 5 \\
T1-weighted gradient-echo in-phase and out-of-phase & Axial & 5 \\
Diffusion-weighted (b values of 0, 500, 1,000 sec/ $\left./ \mathrm{mm}^{2}\right)$ & Axial & 5 \\
T1-weighted 3D fat-suppressed gradient-echo pre- and post-contrast injection & Axial & 3 \\
\hline
\end{tabular}

$\mathrm{MRI}$, magnetic resonance imaging.

imaging, so implicating that CSI methods are not suitable to determine such lesions. Some lesions as adrenocortical carcinoma (ACC), pheochromocytoma, and clear cell renal cell cancer (CCRCC) metastasis show, sometimes, signal loss on out-of-phase images mimicking benign conditions. Quantitative analysis of the chemical phenomenon is provided by calculation of the adrenal-to-spleen CSI ratio or of the signal intensity index (SII) (3). It is reported that the use of diffusion weighted imaging (DWI) is not useful to distinguish adenomas from non-adenomas because of significant overlap in quantitative apparent diffusion coefficient (ADC) values (4-6). Some studies evaluate dynamic gadolinium-enhanced MRI for distinguishing metastases from adenomas showing some success although a validation by other groups is necessary (7). Dynamic contrast-enhanced imaging (DCI) is a useful modality for differentiating adenoma from malignant lesions. The typical enhancement pattern of most adenomas is an early and uniform enhancement on the arterial phase and a rapid washout on the venous phase. Contrarily, most malignant lesions show poor enhancement on the arterial phase and peripheral or heterogeneous enhancement on the venous phase. Even though DCI alone is not more sensitive than CSI for the characterization of adenoma, it is an important tool for outlining adenomas which are not well characterized by CSI (8). A possibility of overcoming the limitations of morphological image in cases of low-fat adenomas, metastases and heterogeneous masses is magnetic resonance spectroscopy (MRS), a noninvasive technique with which is possible to monitor either acute or chronic stages of a disease (9). Analyzing the choline-to-creatinine ratio, MRS allows to differentiate adenoma from non-adenoma (9). However, MRS is not performed widely because its spectral quality is not enough to draw solid conclusions so that further studies will be needed (Table 1) (10).

\section{Normal anatomy}

The adrenal glands are located in the perirenal space, immediately anterosuperior to the upper pole of the kidneys, with an inverted $\mathrm{V}$ or $\mathrm{Y}$ shape (11) and dimensions between 2 to $6 \mathrm{~mm}$ in thickness and 2 to $4 \mathrm{~cm}$ in length. They receive blood supply from the superior, middle and inferior suprarenal arteries, which originate respectively from the inferior phrenic arteries, abdominal aorta and renal arteries $(12,13)$. The venous outflow is different for the two adrenal glands: the right adrenal vein ends in the vena cava, and the left adrenal vein ends in the left renal vein or in the inferior phrenic vein. The gland is composed of an outer cortex, divided into three zones (glomerulosa, fasciculata and reticularis) and a thinner, inner medulla.

\section{Pathological findings}

Depending on their biological behaviour, adrenal glands lesions can be classified into benign and malignant (primary or metastatic) conditions.

\section{Benign conditions}

Adrenocortical adenoma: The most common adrenal tumor (14), in most of the cases is nonfunctional and develops in the cortex. If there is a functional adenoma hypercortisolism (or Cushing syndrome) and primary hyperaldosteronism (or Conn's syndrome) can coexist. The morphologic appearance is a solid tumor, usually small in size $(<4 \mathrm{~cm})$, with hemorrhagic or cystic component, and occasionally calcifications. Because of the intracellular lipid, adenomas can be classified in lipid-rich and lipidpoor (Figure 1). As a result of the different precession frequencies of protons in water molecules and fat, on MRI many adenomas show high signal on in-phase imaging and 

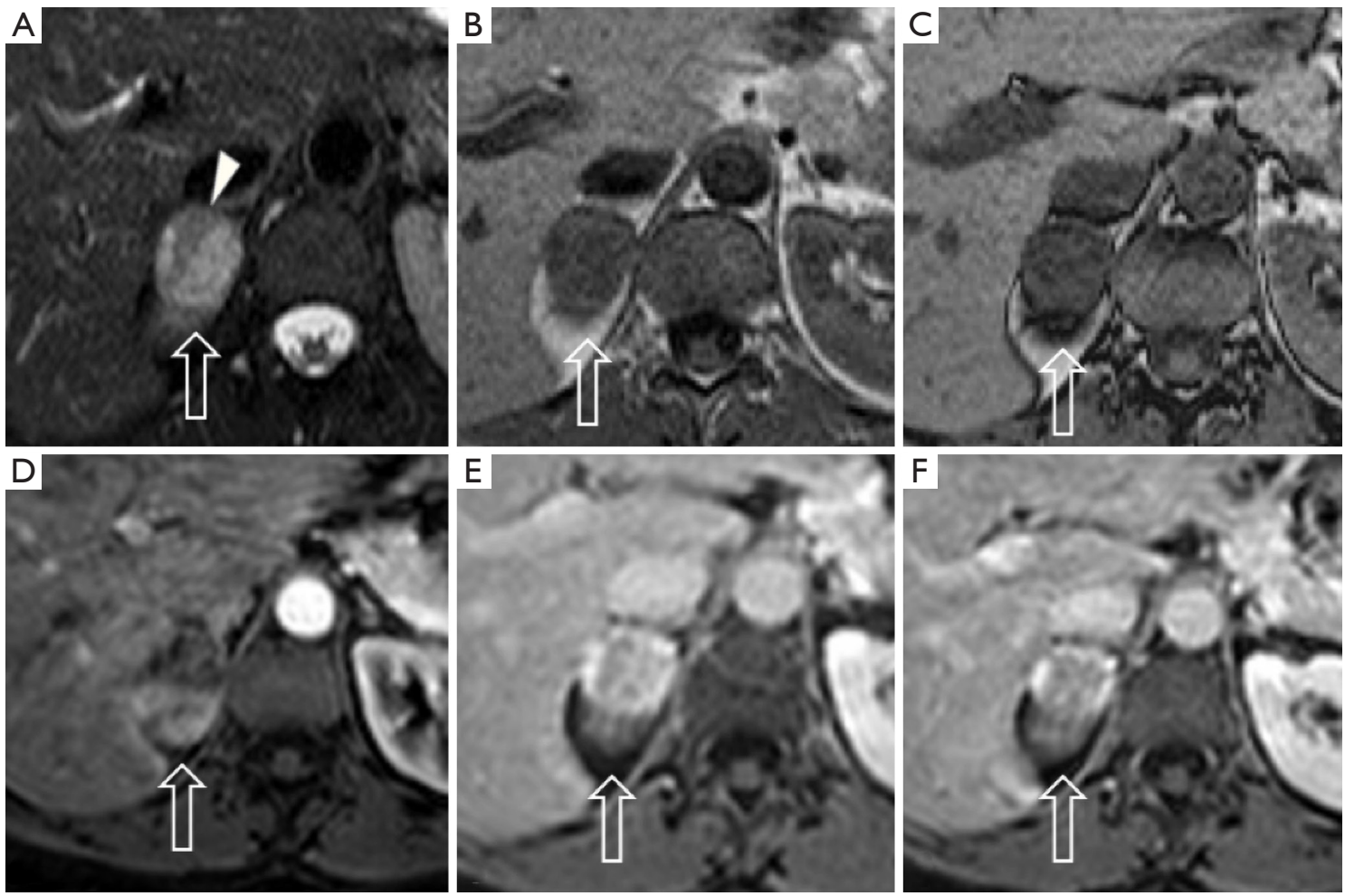

Figure 1 Right adrenal gland lipid-poor adenoma (arrows) in a 51-year-old woman with recent diagnosis of lung cancer. Axial TSE T2-w fat-sat images (A) demonstrate a moderate hyperintense lesion with some areas of signal hypointensity (arrowhead), consistent with the diagnosis of lipid poor adenoma containing a small macroscopic fat component. In agreement with this diagnosis, in-phase (B) and out-ofphase (C) sequences do not demonstrate significant signal loss. Arterial (D) post-contrast phase demonstrates mild dishomogeneous early enhancement with relative wash-out on venous $(\mathrm{E})$ and late venous $(\mathrm{F})$ phases.
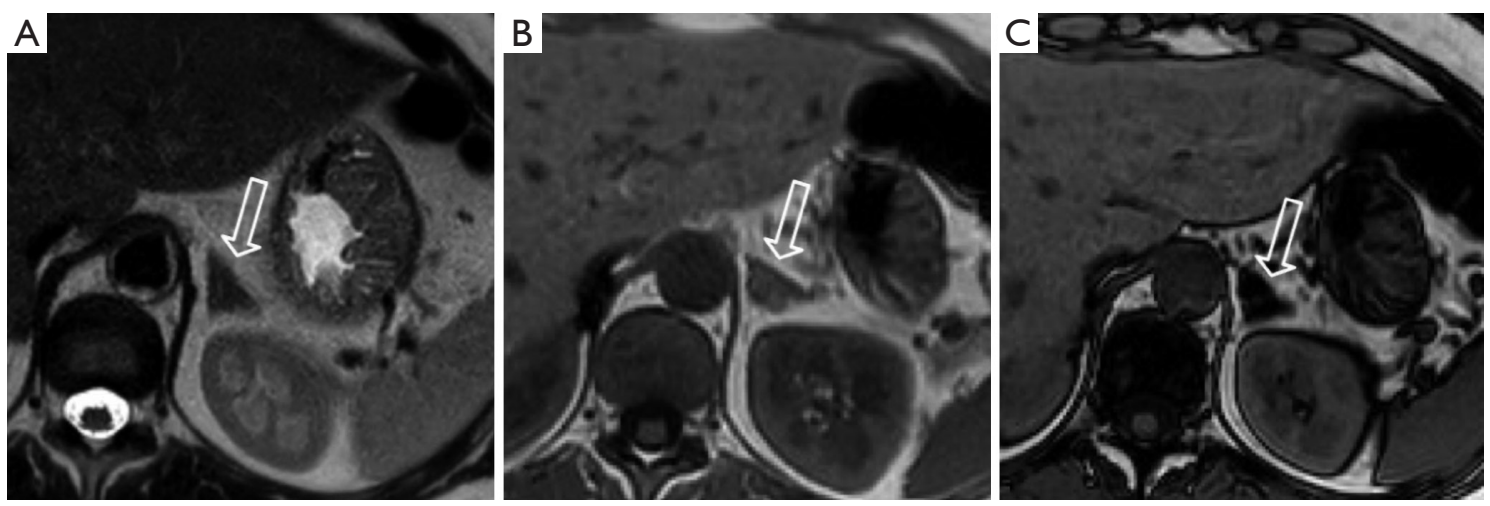

Figure 2 Left adrenal gland macronodular hyperplasia (arrows) in a 46-year-old woman. The lesion appears hypointense on both TSE T2-w sequence (A) and in-phase sequence (B), with a significant signal loss on the out-of-phase sequence (C), coherent with the presence of intracellular fat.

the signal decreases in the out-of-phase imaging. Lipidrich adenomas, in particular, show high intensity signal on T1- and T2-weighted images. After contrast injection most of adenomas show relatively regular enhancement on immediate gadolinium-enhanced images (15).

Hyperplasia: it can be diffuse, characterized by homogeneous thickening of the whole gland, or nodular (Figure 2) and it is typically bilateral (16). Most occur in 

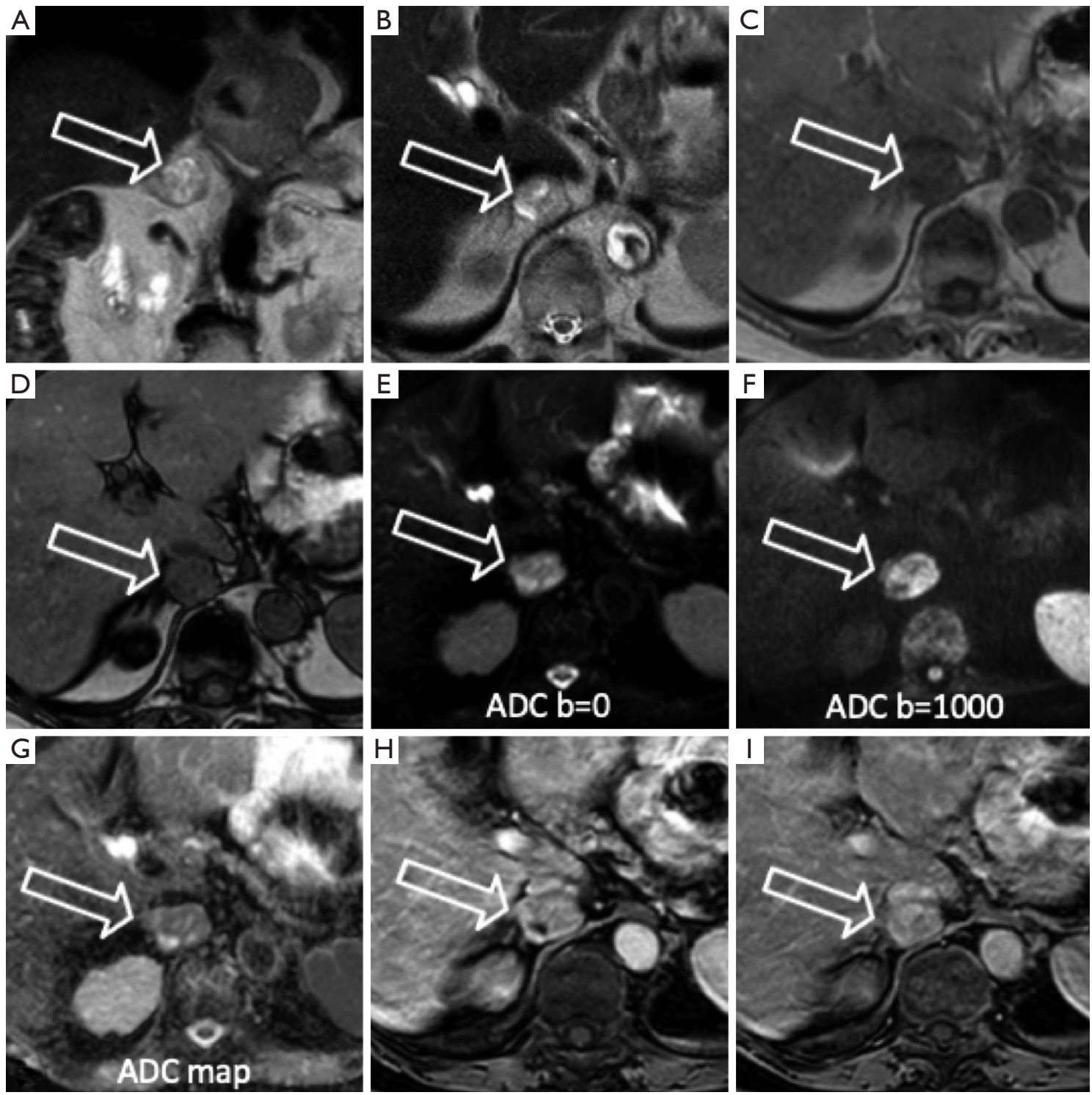

Figure 3 Right adrenal gland pheochromocytoma in a 90-year-old woman. The MRI shows a $32 \times 21 \mathrm{~mm}$ solid tissue (arrows), with an irregular shape, directly in contact with the inferior vena cava and the right diaphragmatic crus. The lesion appears hyperintense on coronal (A) and axial (B) plane TSE T2-w sequences, with no significant signal loss between in-phase (C) and out-of-phase (D) sequences. DWI sequences $(\mathrm{E}, \mathrm{F})$ and $\mathrm{ADC}$ map $(\mathrm{G})$ show restricted diffusion on $\mathrm{b}=1,000$ and ADC map images. After contrast medium injection inhomogeneous enhancement is visible both on arterial $(\mathrm{H})$ and venous (I) phases. DWI, diffusion weighted imaging; ADC, apparent diffusion coefficient.

women (1:4 man vs. women ratio). On MRI the signal is the same of the normal adrenal gland (17).

Pheochromocytoma: known as the $10 \%$ tumor $(10 \%$ non-functioning, $10 \%$ occur in children, $10 \%$ located outside the adrenal gland, $10 \%$ bilateral, $10 \%$ malignant), is a neural crest cell tumor occurring in the adrenal medulla (Figures 3,4).
Usually larger than adenomas may appear as a welldefined mass, solid or cystic to variable degrees (18). Pheochromocytomas do not contain a substantial amount of cytoplasmatic lipid and on MRI maintain their signal intensity on out-of-phase gradient echo (GRE) chemical shift images (Figures 3,4). The use of a quantitative T2-weighted signalintensity ratio could be an important tool to distinguish 

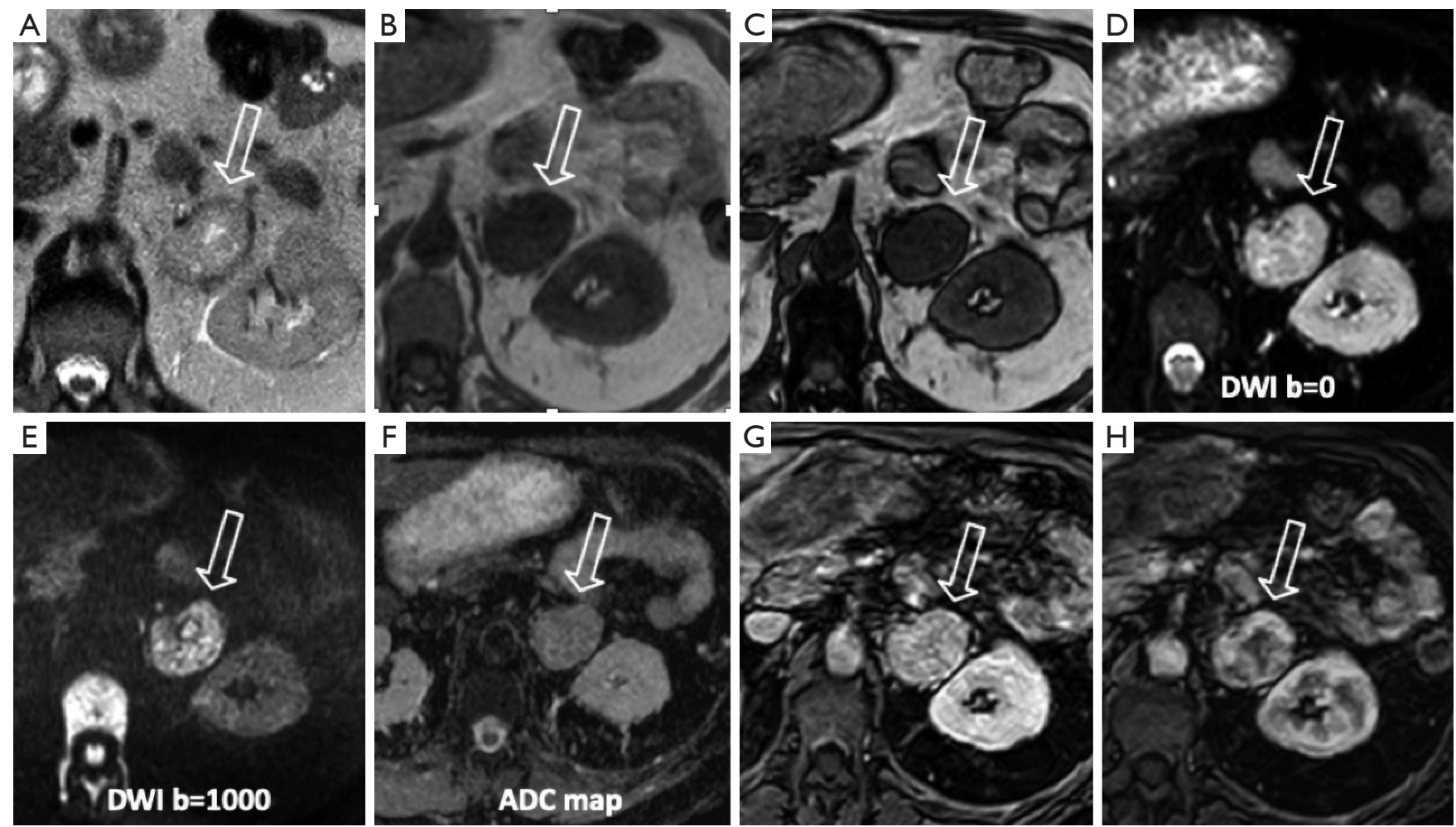

Figure 4 A 70-year-old man with uncontrolled hypertension despite medical therapy with left adrenal gland pheochromocytoma confirmed by circulating catecholamines dosage. The lesion (arrows) presents moderate signal intensity on axial TSE T2-w images (A) and hypointense on in-phase T1 sequence (B) without significant loss of signal in out-of-phase sequence (C) since pheochromocytoma does not contain significant amount of intracellular lipid. The DWI sequences (D,E) in $b=1,000$ images and the ADC map (F) show significant restricted diffusion. Arterial $(\mathrm{G})$ and venous phase $(\mathrm{H})$ demonstrate inhomogeneous enhancement of the lesion with central area of necrosis after Gadolinium administration. DWI, diffusion weighted imaging.

pheochromocytoma from adenoma, due to the presence of intracytoplasmic lipid that definitely excludes the diagnosis of pheochromocytoma $(19,20)$. On MRI the appearance is heterogeneous, because of hemorrhage and necrosis: in 70\% of cases it shows hyperintensity on T2-wheighted images (light bulb sign) $(12,21)$. In the other $30 \%$ pheochromocytomas show moderate or low T2-weighted signal intensity and looking alike to other adrenal diseases $(15,22)$.

Myelolipoma: is a relatively rare benign adrenal neoplasm composed of hematopoietic tissue and mature adipose tissue. On MRI the diagnosis is established by showing macroscopic fat at CSI and fat-suppression MRI. On the basis of MRI features, it's possible to differentiate myelolipomas into three groups: (I) homogeneous, characteristic of lesions predominantly composed of fat; (II) heterogeneous, characteristic of lesion composed by mixed fatty and myeloid elements; (III) nodules, mass-like areas primarily composed of myeloid cells (23). Myelolipomas usually show high signal intensity on T1- and T2-weighted images and a loss of signal of fat-saturated images.

Cyst: relatively rare, often unilateral. There are four types of cyst: endothelial, epithelial, parasitic and pseudocyst $(17,18)$. Normally it looks like a spherical mass with a distinct border often with thin walls, internal septa and calcifications $(13,24)$. On MRI show high signal on T2weighted images and low signal on T1-weighted images, and no contrast enhancement after mdc injection.

Cystic lymphangioma: this is a sort of adrenal endothelial cyst (25), a congenital malformation formed by the benign proliferation of primitive lymphatic vessel. On MRI are typically $\mathrm{T} 1$ hypointense and $\mathrm{T} 2$ hyperintense.

Teratoma: teratomas are solid neoplasm originating from germ cells, with the possibility to differentiate into somatic cells. The typical appearance includes calcifications, hair, teeth, bone, soft tissue and fat. On MRI, T1- and T2-weighted images shows mixed signals. After contrast 

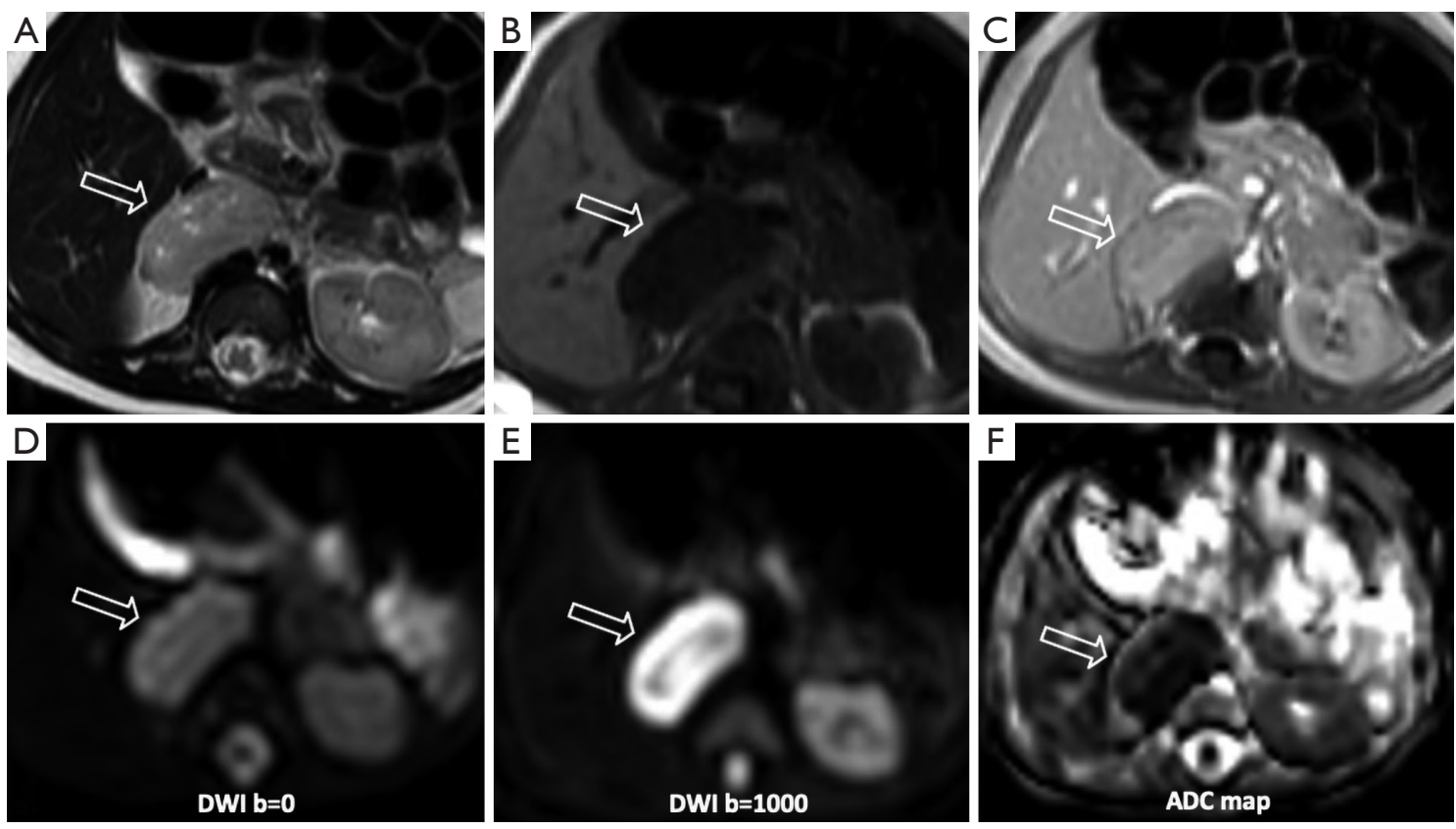

Figure 5 A newborn boy with a right adrenal neuroblastoma incidentally found during an abdominal sonography for persistent oesophageal reflux. The mass (arrows) appears as an inhomogeneous lesion with moderate signal intensity in axial TSE T2-w image (A) and hypointense in T1-w (B), with mild contrast enhancement in arterial phase (C). On DWI sequences (D,E) and ADC map (F), the lesion shows very significant restricted diffusion on $\mathrm{b}=1,000$ images. DWI, diffusion weighted imaging; ADC, apparent diffusion coefficient.

injection it show slight enhancement of the solid component and in particular a significant enhancement of internal septations and of the peripheral rim.

Hemorrhage: it can be caused by traumas or nontraumatic causes, usually has a round or oval appearance and a signal on MRI depending on the stage of the disease. Early hemorrhage is isointense on T1-weighted sequences and hypointense on T2-weighted images. Late hemorrhage shows low signal intensity on T1- and T2-weighted images.

Hemangioma: hemangiomas are classified into 4 types: cavernous, venous, capillary type and mixed (26). They are mainly composed of blood vessels and highly vascularized. Typically, on MRI, shows low signal intensity on T1weighted images and high signal on T2-weighted images.

Ganglioneuroma: rare benign neurogenic tumors originating from the adrenal medulla, appear like solid masses, usually with clear borders and show high signal on T1-weighted images and varied signal on T2-weighted images.

\section{Malignant conditions}

Neuroblastoma: it is the second most frequent abdominal mass in children, usually occurring in the adrenal medulla (Figure 5) (27). Typically characterized by calcifications, necrotic component, hemorrhagic and cystic changes. On MRI has nonhomogeneous appearance and shows hyperintensity on $\mathrm{T} 2$-weighted images and hypointensity on T1-weighted images, with heterogeneous enhancement after mdc injection.

Metastases: the adrenal glands are a common site for metastatic disease (Figures 6,7), most frequently as a consequence of a primary tumor involving lungs, breast, stomach, liver and pancreas. Due to the adrenal blood supply, the most frequent metastatic involvement is hematogenous $(28,29)$. Metastases have no specific imaging findings, usually they appear hypointense on T1-weighted images and hyperintense on T2-weighted images, with ring or irregular enhancement after contrast injection. The use of CSI is limited when metastases from primary malignancies have a lipid or fatty intracellular component, for example clear renal cell carcinoma and hepatocellular carcinoma.

ACC: ACC is a rare, aggressive tumor occurring in the adrenal cortex, usually presenting as a large mass with necrosis and cystic degeneration. On MRI shows a heterogeneous signal, usually high on T2-weighted images 

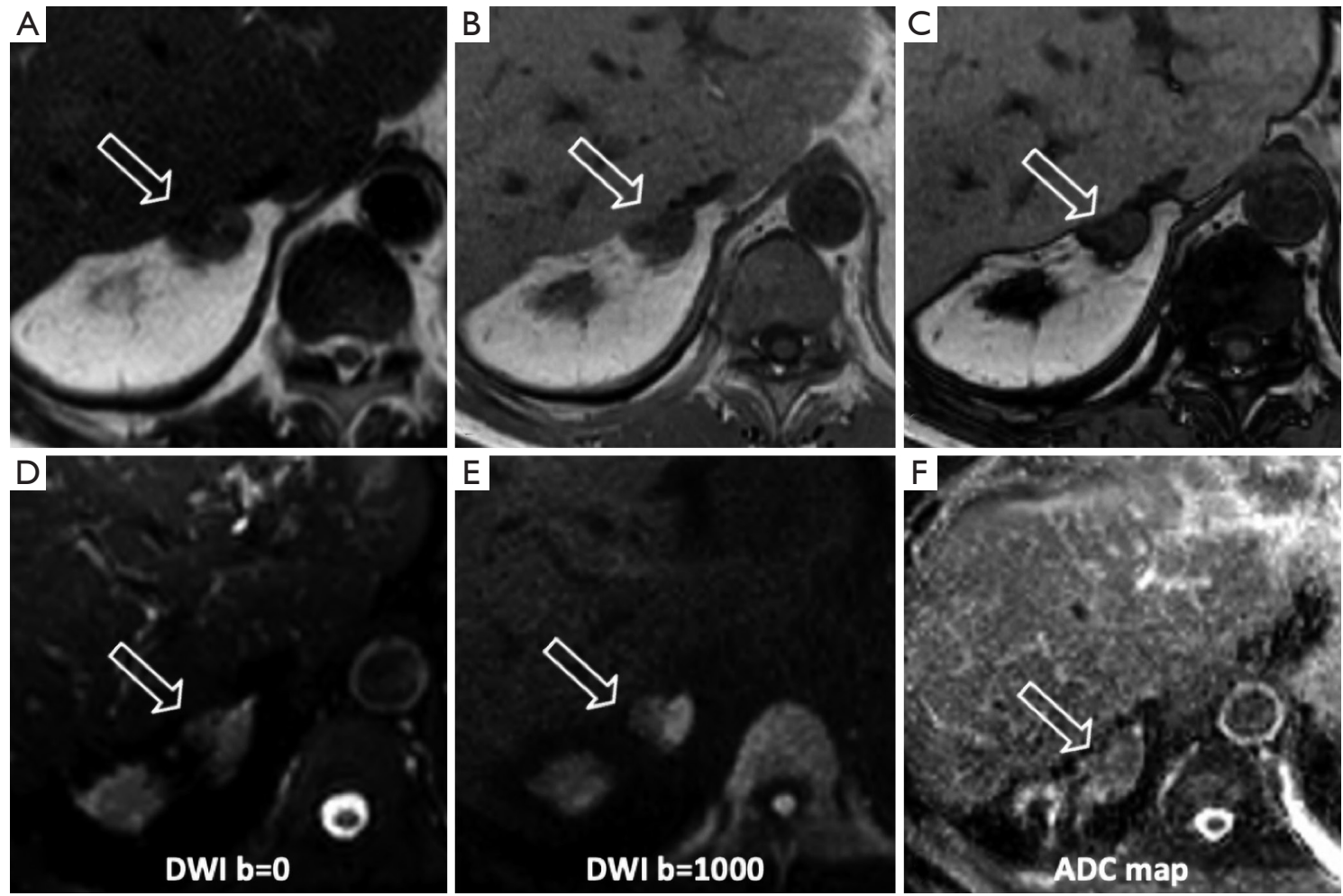

Figure 6 A 70-year-old man with left adrenal metastasis from colon cancer. The lesion (arrows) shows signal hypointensity on the axial TSE T2-w (A) sequence without significant signal difference between the in-phase (B) and out-of-phase (C) sequences. Restricted diffusion is evident on b=1,000 DWI (D,E) sequences and ADC map (F). DWI, diffusion weighted imaging; ADC, apparent diffusion coefficient.

and low on T1-weighted images. Hemorrhagic products, principally methemoglobin, can result in areas of high signal intensity on T1-weighted images; areas of necrosis have high signal intensity on T2-weighted images (Figure 8). A common complication is the venous invasion, frequently of the inferior vena cava.

Lymphoma: the involvement of adrenal gland by lymphoma is infrequent. Primary adrenal lymphoma is extremely rare, usually confined to a single organ, otherwise it is thought to be secondary. Sometimes lymphoma can maintain the gland's adreniform shape and mimic, in its initial stage, adrenal hypertrophy or hyperplasia. Lymphoma lesions are typically large, heterogeneous because of hemorrhagic, necrotic and cystic changes. Typical MRI features are hypointensity on T1-weighted images and hyperintensity on T2-weighted images.

\section{Conclusions}

MR is a very useful imaging technique for adrenal gland lesion assessment, is considered the first choice of imaging method for children, pregnant women, young people and breast-feeding mothers (30).

Although CSI is accurate to distinguish adenomas from metastatic disease of the adrenal glands, false-positive findings of benignancy can occur in cases of metastatic disease, specially related to clear cell renal cell carcinoma and hepatocellular carcinoma (31).

The use of DWI is not yet considered a valid method to differentiate adenomas from nonadenomas because of conflicting results reported in literature.

Spectroscopy even though is a promising technique needs more studies to be validated. 

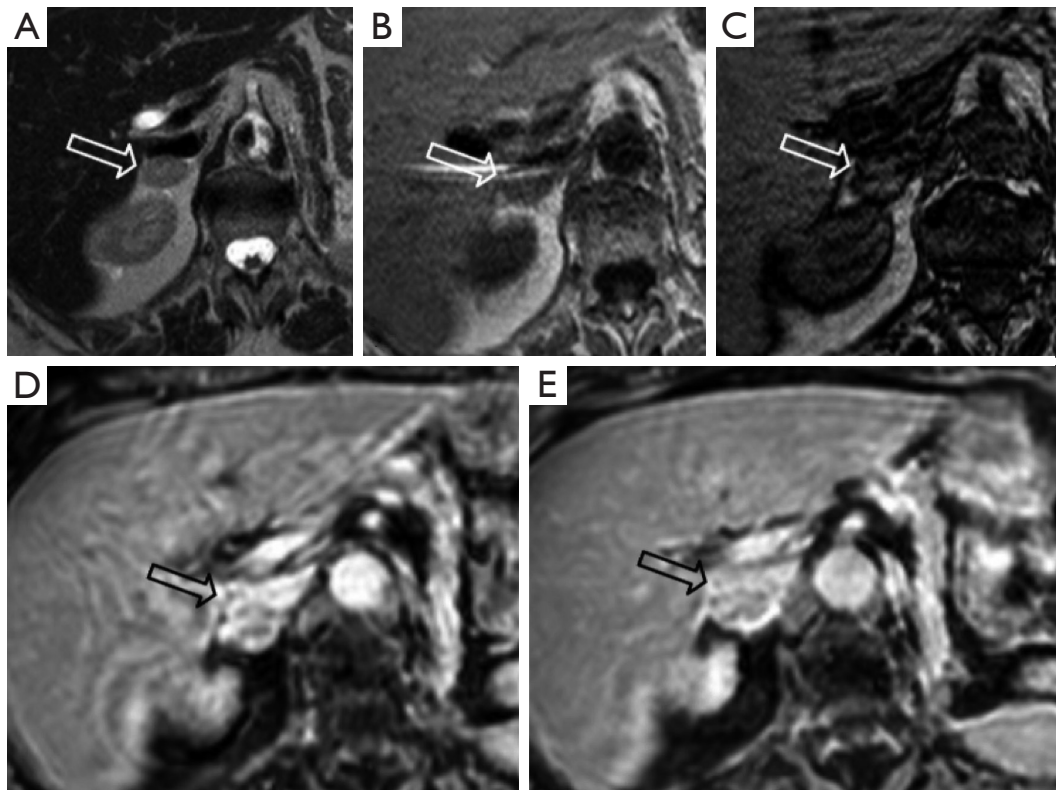

Figure 7 Right adrenal gland metastases in an 81-year-old woman with history of left papillary renal cell carcinoma. The lesion (arrows) shows mild signal intensity on TSE T2w images (A), in-phase (B) and out-of-phase (C) sequences. The lesion enhances significantly after contrast medium injection (D), without significant wash-out in venous phase (E).
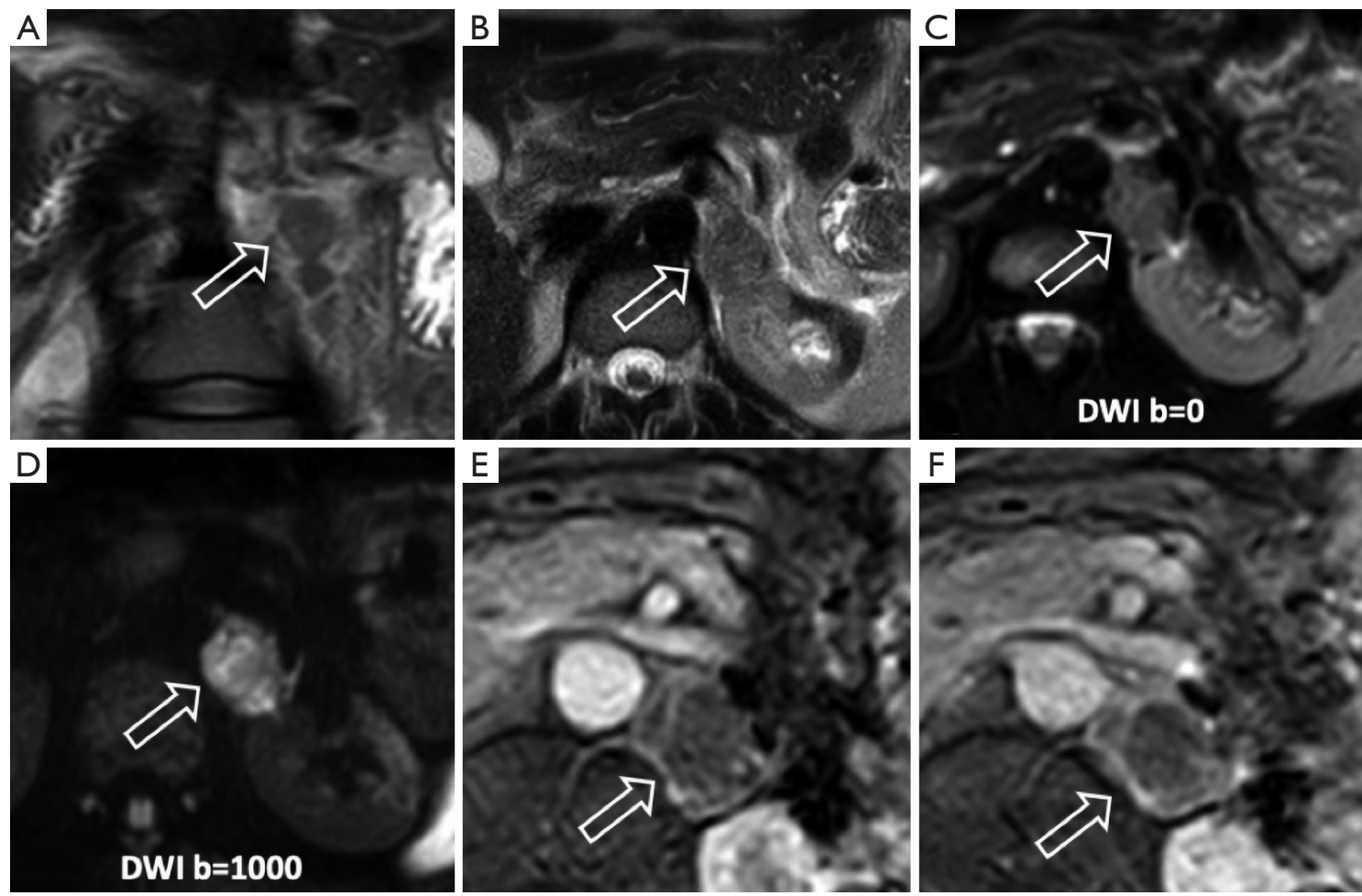

Figure 8 A 32-year-old woman with left adrenal cortex carcinoma (arrows). TSE T2-w sequences on the coronal (A) and axial (B) plane demonstrates an oval shaped lesion with moderate signal intensity of the left adrenal gland. DWI sequences (C,D) show significant restricted diffusion on $b=1,000$ images. On arterial $(\mathrm{E})$ and venous $(\mathrm{F})$ phases the mass shows inhomogeneous peripheral enhancement, related to the presence of central foci of necrotic tissue. DWI, diffusion weighted imaging. 


\section{Acknowledgments}

None.

\section{Footnote}

Conflicts of Interest: The authors have no conflicts of interest to declare.

\section{References}

1. Elsayes KM, Mukundan G, Narra VR, et al. Adrenal masses: MR imaging features with pathologic correlation. RadioGraphics 2004;24:S73-86.

2. Adam SZ, Nikolaidis P, Horowitz JM, et al. Chemical Shift MR Imaging of the Adrenal Gland: Principles, Pitfalls, and Applications. RadioGraphics 2016;36:414-32.

3. Hood MN, Ho VB, Smirniotopoulos JG, et al. Chemical shift: the artifact and clinical tool revisited. RadioGraphics 1999;19:357-71.

4. Tsushima Y, Takahashi-Taketomi A, Endo K. Diagnostic utility of diffusion-weighted MR imaging and apparent diffusion coefficient value for the diagnosis of adrenal tumors. J Magn Reson Imaging 2009;29:112-7.

5. Sandrasegaran K, Patel AA, Ramaswamy R, et al. Characterization of adrenal masses with diffusion-weighted imaging. AJR Am J Roentgenol 2011;197:132-8.

6. Halefoglu AM, Altun I, Disli C, et al. A prospective study on the utility of diffusion-weighted and quantitative chemical-shift magnetic resonance imaging in the distinction of adrenal adenomas and metastases. J Comput Assist Tomogr 2012;36:367-74.

7. Becker-Weidman D, Kalb B, Mittal PK, et al. Differentiation of lipid-poor adrenal adenomas from non-adenomas with magnetic resonance imaging: utility of dynamic, contrast enhancement and single-shot T2weighted sequences. Eur J Radiol 2015;84:2045-51.

8. Rodacki K, Ramalho M, Dale BM, et al. Combined chemical shift imaging with early dynamic serial gadolinium-enhanced MRI in the characterization of adrenal lesions. AJR Am J Roentgenol 2014;203:99-106.

9. Melo HJ, Goldman SM, Szejnfeld J, et al. Application of a protocol for magnetic resonance spectroscopy of adrenal glands: An experiment with over 100 cases. Radiol Bras 2014;47:333-41.

10. Blake MA, Cronin CG, Boland GW. Adrenal imaging. AJR Am J Roentgenol 2010;194:1450-60.

11. Korivi BR, Elsayes KM, de Castro SF, et al. An Update of
Practical CT Adrenal Imaging: What Physicians Need to Know. Current Radiol Rep 2015;3:12.

12. Lattin GE Jr, Sturgill ED, Tujo CA, et al. From the radiologic pathology archives: Adrenal tumors and tumor-like conditions in the adult: radiologic- pathologic correlation. RadioGraphics 2014;34:805-29.

13. Song JH, Mayo-Smith WW. Current status of imaging for adrenal gland tumors. Surg Oncol Clin N Am 2014;23:847-61.

14. Ctvrtlik F, Koranda P, Tichy T. Adrenal disease: a clinical update and overview of imaging. A review. Biomed Pap Med Fac Univ Palacky Olomouc Czech Repub 2014;158:23-34.

15. Mayo-Smith WW, Boland GW, Noto RB, et al. Stateof-the-art adrenal imaging. RadioGraphics 2001;21:9951012 .

16. Wang YX, He GX, Du LJ, et al. CT findings in congenital adrenal hyperplasia due to 11 beta hydroxylase deficiency at puberty age. JBR-BTR 1999;82:11-2.

17. Liu H, Wang AP, Bo YH, et al. CT and MRI Findings of Adrenal Cystic Lesions. J Clin Radiol 2015;34:591-5.

18. Farrugia FA, Martikos G, Surgeon C, et al. Radiology of the adrenal incidentalomas. Review of the literature. Endocr Regul 2017;51:35-51.

19. Schieda N, Alrashed A, Flood TA, et al. Comparison of quantitative MRI and CT washout analysis for differentiation of adrenal pheochromocytoma from adrenal adenoma. AJR 2016;206:1141-8.

20. Borhani AA, Hosseinzadeh K. Quantitative versus qualitative methods in evaluation of T2 signal intensity to improve accuracy in diagnosis of pheochromocytoma. AJR 2015;205:302-10.

21. Munir S, Waseem M. Addison Disease. StatPearls. Treasure Island (FL): StatPearls Publishing StatPearls Publishing LLC., 2018.

22. Blake MA, Kalra MK, Maher MM, et al. Pheochromocytoma: an imaging chameleon. RadioGraphics 2004;24:S87-9.

23. Boraschi P, Braccini G, Gigoni R, et al. Adrenal myelolipomas: their magnetic resonance assessment. Clin Ter 1996;147:549-57.

24. Boland GW, Blake MA, Hahn PF, et al. Incidental adrenal lesions: principles, techniques, and algorithms for imaging characterization. Radiology 2008;249:756-75.

25. Rowe SP, Bishop JA, Prescott JD, et al. CT Appearance of Adrenal Cystic Lymphangioma: Radiologic-Pathologic Correlation. AJR Am J Roentgenol 2016;206:81-5.

26. Secil M, Demir O, Yorukoglu K. MRI of adrenal 
lymphangioma: a case report. Quant Imaging Med Surg 2013;3:347-8.

27. Herr K, Muglia VF, Koff WJ, et al. Imaging of the adrenal gland lesions. Radiol Bras 2014;47:228-39.

28. Lim W, Ridge CA, Nicholson AG, et al. The 8th lung cancer TNM classification and clinical staging system: review of the changes and clinical implications. Quant Imaging Med Surg 2018;8:709-18.

29. Wang YX, Gong JS, Suzuki K, et al. Evidence based imaging strategies for solitary pulmonary nodule. J Thorac

Cite this article as: d'Amuri FV, Maestroni U, Pagnini F, Russo U, Melani E, Ziglioli F, Negrini G, Cella S, Cappabianca S, Reginelli A, Barile A, De Filippo M. Magnetic resonance imaging of adrenal gland: state of the art. Gland Surg 2019;8(Suppl 3):S223-S232. doi: 10.21037/gs.2019.06.02
Dis 2014;6:872-87.

30. Caoili EM, Korobkin M, Francis IR, et al. Delayed enhanced CT of lipid-poor adrenal adenomas. AJR Am J Roentgenol 2000;175:1411-5.

31. Sydow BD, Rosen MA, Siegelman ES. Intracellular lipid within metastatic hepatocellular carcinoma of the adrenal gland: a potential diagnostic pitfall of chemical shift imaging of the adrenal gland. AJR Am J Roentgenol 2006;187:W550-1. 